\title{
Reconstruction of Decomposable Discrete Sets from Four Projections
}

\author{
Péter Balázs \\ Department of Informatics, University of Szeged, \\ Árpád tér 2, H-6720 Szeged, Hungary \\ pbalazs@inf.u-szeged.hu
}

\begin{abstract}
In this paper we introduce the class of decomposable discrete sets and give a polynomial algorithm for reconstructing discrete sets of this class from four projections. It is also shown that the class of decomposable discrete sets is more general than the class $\mathcal{S}_{8}^{\prime}$ of $h v$-convex 8but not 4-connected discrete sets which was studied in 3 . As a consequence we also get that the reconstruction from four projections in $\mathcal{S}_{8}^{\prime}$ can be solved in $O(m n)$ time.
\end{abstract}

Keywords: discrete tomography, reconstruction from projections, decomposable discrete set

\section{Introduction}

One of the most frequently studied problems in the area of discrete tomography [14, 15] is the reconstruction of 2-dimensional discrete sets from few (usually up to four) projections. Several theoretical questions are connected with reconstruction such as existence and uniqueness (as a summary see [6, 9, 12, ). There are also reconstruction algorithms for different classes of discrete sets (e.g., [4, 5, 7, 8, 11, 16, 17, 19]). However, the reconstruction problem is usually underdetermined and the number of solutions can be very large. Moreover, the reconstruction in certain classes can be NP-hard (see [21]). In order to keep the reconstruction process tractable and to reduce the number of solutions a commonly used technique is to suppose having some a priori information of the set to be reconstructed. The most frequently used properties are connectedness, directedness and some kind of discrete versions of the convexity. In this paper we introduce a new property of discrete sets, namely the decomposability, and study uniqueness and reconstruction problems in the class of discrete sets having this property.

This article is structured as follows. First, the necessary definitions are introduced in Section 2. In Section 3 we define the class of decomposable discrete sets and give a polynomial algorithm for reconstructing sets belonging to this class using four projections. In Section 4 we show that every 8 - but not 4 -connected set is decomposable and applying the results of Section 3 we get an $O(m n)$ algorithm for the reconstruction problem in this class using four projections. Finally, in Section 5 we conclude our results. 


\section{Definitions and Notation}

Let $\hat{F}=\left(\hat{f}_{i j}\right)_{m \times n}$ be a binary matrix where $m, n \geq 1$. Let $F$ denote the set of positions $(i, j)$ where $\hat{f}_{i j}=1$, i.e., $F=\left\{(i, j) \mid \hat{f}_{i j}=1\right\} . F$ is called a discrete set, its elements are called points or positions. The $k$-th diagonal/antidiagonal $(k=1, \ldots, m+n-1)$ of $\hat{F}$ are defined by the set $D_{k} / A_{k}$, respectively, where

$$
\begin{gathered}
D_{k}=\{(i, j) \in\{1, \ldots, m\} \times\{1, \ldots, n\} \mid i+(n-j)=k\}, \\
A_{k}=\{(i, j) \in\{1, \ldots, m\} \times\{1, \ldots, n\} \mid i+j=k+1\} .
\end{gathered}
$$

Let $\mathcal{F}$ denote the class of discrete sets. For any discrete set $F \in \mathcal{F}$ we define the functions $\mathcal{H}, \mathcal{V}, \mathcal{D}$, and $\mathcal{A}$ as follows.

$\mathcal{H}: \mathcal{F} \longrightarrow \mathbb{N}_{0}^{m}, \mathcal{H}(F)=H=\left(h_{1}, \ldots, h_{m}\right)$, where

$$
h_{i}=\sum_{j=1}^{n} \hat{f}_{i j}, \quad i=1, \ldots, m,
$$

$\mathcal{V}: \mathcal{F} \longrightarrow \mathbb{N}_{0}^{n}, \mathcal{V}(F)=V=\left(v_{1}, \ldots, v_{n}\right)$, where

$$
v_{j}=\sum_{i=1}^{m} \hat{f}_{i j}, \quad j=1, \ldots, n
$$

$\mathcal{D}: \mathcal{F} \longrightarrow \mathbb{N}_{0}^{m+n-1}, \mathcal{D}(F)=D=\left(d_{1}, \ldots, d_{m+n-1}\right)$, where

$$
d_{k}=\sum_{(i, j) \in D_{k}} \hat{f}_{i j}=\left|F \cap D_{k}\right|, \quad k=1, \ldots, m+n-1,
$$

$\mathcal{A}: \mathcal{F} \longrightarrow \mathbb{N}_{0}^{m+n-1}, \mathcal{A}(F)=A=\left(a_{1}, \ldots, a_{m+n-1}\right)$, where

$$
a_{k}=\sum_{(i, j) \in A_{k}} \hat{f}_{i j}=\left|F \cap A_{k}\right|, \quad k=1, \ldots, m+n-1 .
$$

The vectors $H, V, D$ and $A$ are called the row, column, diagonal and antidiagonal projections of $F$, respectively (see Fig. 11). In the following we suppose that $h_{i}>0$ and $v_{j}>0$ for all $i \in\{1, \ldots, m\}$ and $j \in\{1, \ldots, n\}$. The cumulated horizontal/vertical/diagonal/antidiagonal vectors are denoted by $\widetilde{H}=\left(\widetilde{h}_{1}, \ldots, \widetilde{h}_{m}\right)$, $\widetilde{V}=\left(\widetilde{v}_{1}, \ldots, \widetilde{v}_{n}\right), \widetilde{D}=\left(\widetilde{d}_{1}, \ldots, \widetilde{d}_{m+n-1}\right)$, and $\widetilde{A}=\left(\widetilde{a}_{1}, \ldots, \widetilde{a}_{m+n-1}\right)$, respectively, and defined by the following formulas (see Fig. 1)

$$
\begin{aligned}
& \widetilde{h}_{i}=\sum_{l=1}^{i} h_{l}, \quad i=1, \ldots, m, \\
& \widetilde{v}_{j}=\sum_{l=1}^{j} v_{l}, \quad j=1, \ldots, n,
\end{aligned}
$$




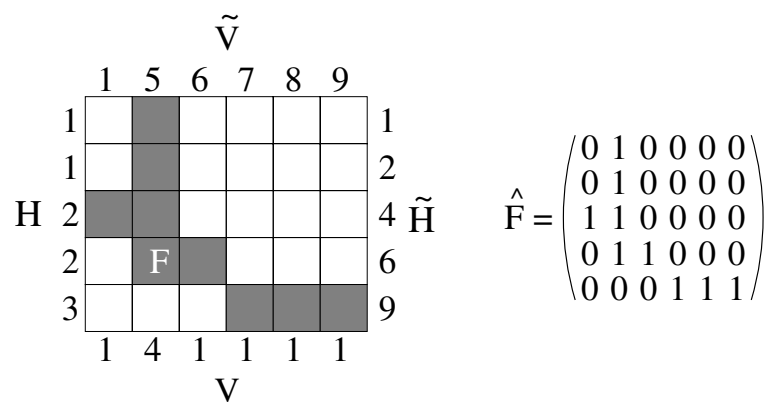

Fig. 1. An $h v$-convex 8- but not 4-connected discrete set $F$ and the corresponding binary matrix $\hat{F}$. The elements of $F$ are marked with grey squares. The projections of $F$ are the vectors $H, V, D=(0,0,0,0,2,2,3,2,0,0)$, and $A=$ $(0,1,2,1,1,1,0,1,1,1)$. The cumulated vectors are $\widetilde{H}, \widetilde{V}, \widetilde{D}=(0,0,0,0,2,4,7,9,9,9)$, and $\widetilde{A}=(0,1,3,4,5,6,6,7,8,9)$

$$
\widetilde{d}_{k}=\sum_{l=1}^{k} d_{l}, \quad \widetilde{a}_{k}=\sum_{l=1}^{k} a_{l}, \quad k=1, \ldots, m+n-1 .
$$

Given a class $\mathcal{G} \subseteq \mathcal{F}$ of discrete sets, we say that the discrete set $F \in \mathcal{G}$ is unique in the class $\mathcal{G}$ (with respect to some projections) if there is no different discrete set $F^{\prime} \in \mathcal{G}$ with the same projections.

Two points $P=\left(p_{1}, p_{2}\right)$ and $Q=\left(q_{1}, q_{2}\right)$ in a discrete set $F$ are said to be 4-adjacent if $\left|p_{1}-q_{1}\right|+\left|p_{2}-q_{2}\right|=1$. The points $P$ and $Q$ are said to be 8 -adjacent if they are 4-adjacent or $\left|p_{1}-q_{1}\right|=1$ and $\left|p_{2}-q_{2}\right|=1$. The sequence of distinct points $P_{0}, \ldots, P_{k}$ is a $4 / 8$-path from point $P_{0}$ to point $P_{k}$ in a discrete set $F$ if each point of the sequence is in $F$ and $P_{l}$ is $4 / 8$-adjacent, respectively, to $P_{l-1}$ for each $l=1, \ldots, k$. Two points are $4 / 8$-connected in the discrete set $F$ if there is a 4/8-path, respectively, in $F$ between them. A discrete set $F$ is 4/8-connected if any two points in $F$ are 4/8-connected, respectively, in $F$. The 4 -connected set is also called as polyomino. The discrete set $F$ is horizontally convex/vertically convex (or shortly, $h$-convex/v-convex) if its rows/columns are 4 -connected, respectively. The $h$ - and $v$-convex sets are called $h v$-convex (see Fig. 1). In this paper we are going to study the reconstruction problem from four projections in several classes. Given a class $\mathcal{G} \subseteq \mathcal{F}$ the problem can be formulated as follows

\section{4-Reconstruction $(\mathcal{G})$.}

Instance: Four non-negative vectors $H \in \mathbb{N}^{m}, V \in \mathbb{N}^{n}, D \in \mathbb{N}_{0}^{m+n-1}$ and $A \in \mathbb{N}_{0}^{m+n-1}$.

Task: $\quad$ Construct a discrete set $F \in \mathcal{G}$ with $\mathcal{H}(F)=H, \mathcal{V}(F)=V$, $\mathcal{D}(F)=D$ and $\mathcal{A}(F)=A$. 


\section{Reconstruction of Decomposable Discrete Sets}

Let $F$ be a discrete set. A maximal 4-connected subset of $F$ is called a component of $F$ (e.g., in Fig. 1 there are two components: $\{(5,4),(5,5),(5,6)\}$ and $\{(1,2),(2,2),(3,1),(3,2),(4,2),(4,3)\})$. Clearly, the components of $F$ give a (uniquely determined) partition of $F$. We will use the concept of smallest containing discrete rectangle of $F$ (SCDR) which corresponds to the notion of strong convex hull of $F$ (see [20]). Throughout this paper we always study discrete sets having the following properties

$(\alpha)$ the components are uniquely reconstructible from their horizontal and vertical projections in polynomial time, and

$(\beta)$ the sets of the row/column indices of the components are disjoint, i.e., if $I \times J \subseteq\{1, \ldots, m\} \times\{1, \ldots, n\}$ is the SCDR of a component of the discrete set $F$, then $\bar{I} \times J \cap F=I \times \bar{J} \cap F=\emptyset$ (where $\bar{I}=\{1, \ldots, m\} \backslash I$ and $\bar{J}=\{1, \ldots, n\} \backslash J)$.

In fact, to satisfy property $(\alpha)$ we need to have some a priori information about the components. For example, NW-directed $h v$-convex discrete sets can be used as components since in this class property $(\alpha)$ is fulfilled 10 .

The NorthWest-gluing (or shortly, NW-gluing) is an operator defined by

$$
\mathcal{F}^{2} \longrightarrow \mathcal{F}: C \times D \rightarrow F, \text { where } \hat{F}=\left(\begin{array}{cc}
\hat{C} & \mathbf{0} \\
\mathbf{0} & \hat{D}
\end{array}\right)
$$

If $C$ is a single component then we say that $C$ is the $N W$-component of $F$. NE-, SE-, SW-gluings and -components are defined similarly. We say that a discrete set $F$ consisting of $k(k \geq 2)$ components is decomposable if

(i) $F$ satisfies properties $(\alpha)$ and $(\beta)$, and

(ii) if $k>2$ then we get $F$ by gluing a single component to a decomposable discrete set consisting of $k-1$ components using one of the four gluing operators.

As a straight consequence of the definition we get that every discrete set consisting of three components and satisfying properties $(\alpha)$ and $(\beta)$ is decomposable. Figure 2 shows some decomposable and undecomposable configurations if the set consists of four components. The class of decomposable discrete sets is denoted by $\mathcal{D} E C$. The following lemma shows an important property of the decomposable discrete sets.

Lemma 1. Let $F \in \mathcal{D} E C$ having more than two components, $C$ be a component of $F$ with the $S C D R I \times J$. Let $F^{\prime}$ be the discrete set that we get by deleting rows $I \times\{1, \ldots, n\}$ and columns $\{1, \ldots, m\} \times J$ from $F$. Then $F^{\prime} \in \mathcal{D} E C$.

Proof. See [1].

On the basis of properties $(\alpha)$ and $(\beta)$ in the reconstruction of a decomposable discrete set it is sufficient to identify the SCDRs of the components. In order to do this we first give a necessary condition. 


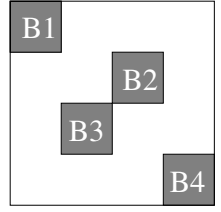

(a)

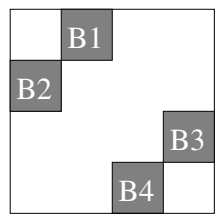

(e)

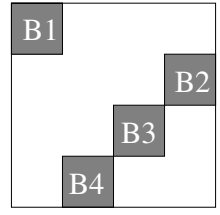

(b)

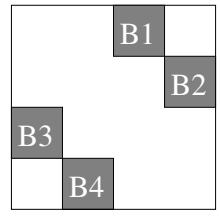

(f)

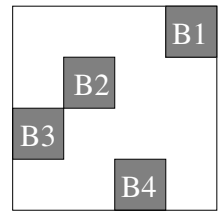

(c)

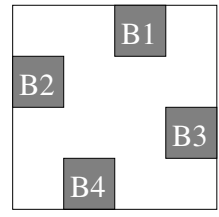

(g)

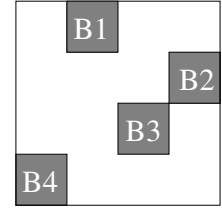

(d)

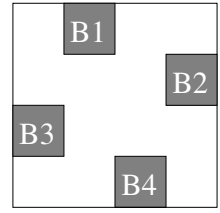

(h)

Fig. 2. Some decomposable (first row) and undecomposable (second row) configurations of the components. The SCDRs of the components are denoted by B1, B2, B3, and B4

Theorem 1. Let $F \in \mathcal{D} E C$. If $(i, j)$ is the bottom right position of the $S C D R$ of the $N W$-component of $F$ then $i$ is the smallest integer for which there exists an integer $j$ such that $\widetilde{h}_{i}=\widetilde{v}_{j}=\widetilde{a}_{i+j-1}$ and $a_{i+j}=0$.

Proof. Define a set $E$ as follows

$$
E=(\{1, \ldots, i\} \times\{j+1, \ldots, n\}) \cup(\{i+1, \ldots, m\} \times\{1, \ldots, j\}) .
$$

If $(i, j)$ is the bottom right position of the SCDR of the NW-component then $F \cap E=\emptyset$ (see Fig. 3), and so

$$
\begin{aligned}
\widetilde{h}_{i}=\sum_{t=1}^{i} h_{t}= & |F \cap\{1, \ldots, i\} \times\{1, \ldots, n\}|=|F \cap\{1, \ldots, i\} \times\{1, \ldots, j\}| \\
& =|F \cap\{1, \ldots, m\} \times\{1, \ldots, j\}|=\sum_{t=1}^{j} v_{t}=\widetilde{v}_{j} .
\end{aligned}
$$

Furthermore, $\left(F \cap A_{k}\right) \cap E \subseteq F \cap E=\emptyset$ for every $k=1, \ldots, m+n-1$ (see again Fig. 3). Then, recalling that $a_{k}=\left|F \cap A_{k}\right|$ for $k=1, \ldots, n+m-1$ we get that

$$
\widetilde{a}_{i+j-1}=\sum_{k=1}^{i+j-1}\left|F \cap A_{k}\right|=|F \cap\{1, \ldots, i\} \times\{1, \ldots, j\}|=\widetilde{h}_{i}=\widetilde{v}_{j} .
$$

Moreover, $A_{i+j} \subseteq E$ (see Fig. [3). Then, $F \cap A_{i+j} \subseteq F \cap E=\emptyset$ and we get that

$$
a_{i+j}=\left|F \cap A_{i+j}\right| \leq|F \cap E|=0 .
$$

Finally, assume that an integer $i^{\prime}<i$ exists for which an integer $j^{\prime}$ exists such that $\widetilde{h}_{i^{\prime}}=\widetilde{v}_{j^{\prime}}=\widetilde{a}_{i^{\prime}+j^{\prime}-1}$ and $a_{i^{\prime}+j^{\prime}}=0$. Clearly, in this case $j^{\prime}<j$. Since 


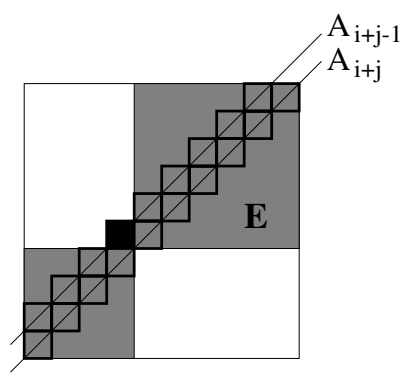

Fig. 3. The relations between the sets $A_{i+j-1}, A_{i+j}$ and $E$. The position $(i, j)$ is marked with black square. The antidiagonals $A_{i+j-1}$ and $A_{i+j}$ are marked with bold squares. The set $E$ is drawn with grey squares.

$(i, j)$ is the bottom right position of the SCDR of the NW-component and every component is a polyomino we get that the 1 st $, \ldots,(i+j)$-th coordinates of the antidiagonal projection have to be of the form $\left(0, \ldots, 0, a_{k_{1}}, \ldots, a_{k_{2}}, 0, \ldots, 0\right)$, where $1 \leq k_{1} \leq k_{2}<i+j$ and $a_{l} \neq 0$ for every $k_{1} \leq l \leq k_{2}$. But then $a_{i^{\prime}+j^{\prime}}=0$ only if $i^{\prime}+j^{\prime}<k_{1}$ or $i^{\prime}+j^{\prime}>k_{2}$. If $i^{\prime}+j^{\prime}<k_{1}$ then $\widetilde{a}_{i^{\prime}+j^{\prime}-1}=0$. Since the cumulated horizontal sums of a discrete set having nonzero rows are always satisfy the relation

$$
0<\widetilde{h}_{1}<\widetilde{h}_{2}<\ldots<\widetilde{h}_{m}
$$

we get that $\widetilde{a}_{i^{\prime}+j^{\prime}-1}<\widetilde{h}_{i^{\prime}}$ which is a contradiction. Otherwise, i.e., if $i^{\prime}+j^{\prime}>k_{2}$ then $\widetilde{h}_{i}>\widetilde{h}_{i^{\prime}}$ (since $i>i^{\prime}$ and (15) holds) and we get that $\widetilde{a}_{i^{\prime}+j^{\prime}-1}=\widetilde{a}_{i+j-1}=$ $\widetilde{h}_{i}>\widetilde{h}_{i^{\prime}}$ which is, again, a contradiction.

Similar theorems can be given for NE-, SE-, and SW-components. Before giving a sufficient condition for finding the SCDR of a component of $F$ we introduce some further concepts. Let $F, F^{\prime} \in \mathcal{F}$ such that $F^{\prime} \backslash F=\left\{\left(p_{1}, q_{1}\right)\right\}$ and $F \backslash F^{\prime}=\left\{\left(p_{2}, q_{2}\right)\right\}$. If $\left(p_{1}+k, q_{1}+k\right)=\left(p_{2}, q_{2}\right)$ for a $k \in \mathbb{Z} \backslash\{0\}$ then we say that we get $F^{\prime}$ by applying a slide on $F$. Similarly, if $\left(p_{1}+k, q_{1}-k\right)=\left(p_{2}, q_{2}\right)$ for a $k \in \mathbb{Z} \backslash\{0\}$ then we say that we get $F^{\prime}$ by applying an antislide on $F$. Clearly, applying slides/antislides on a discrete set, the diagonal/antidiagonal projection does not change, respectively. The following lemma shows an important relation between polyominoes and decomposable discrete sets having the same horizontal, vertical, and antidiagonal projections.

Lemma 2. Let $P$ be a polyomino and $F \in \mathcal{D} E C$ such that $\mathcal{H}(P)=\mathcal{H}(F)$, $\mathcal{V}(P)=\mathcal{V}(F)$, and $\mathcal{A}(P)=\mathcal{A}(F)$. Then, the SCDRs of the components of $F$ are connected to each other with their bottom left and upper right corners.

Proof. See 1] for the proof based on the results of [13].

An analogue of Lemma 2 replacing the antidiagonal projection with the diagonal one can also be proven. In this case the components of the decomposable 
discrete set must be connected to each other with their bottom right and upper left corners. With the aid of the following theorem it is possible to test whether the decomposable discrete set has a NW-component and if so then the component itself can also be reconstructed.

Theorem 2. Let $F \in \mathcal{D} E C, \mathcal{H}(F)=\left(h_{1}, \ldots, h_{m}\right), \mathcal{V}(F)=\left(v_{1}, \ldots, v_{n}\right)$, and $\mathcal{A}(F)=\left(a_{1}, \ldots, a_{m+n-1}\right)$. Moreover, let $(i, j)$ be a position satisfying the necessary conditions of Theorem 1 . If a polyomino $P$ exists according to the a priori information which guarantees that property $(\alpha)$ is satisfied such that $\mathcal{H}(P)=$ $\left(h_{1}, \ldots, h_{i}\right), \mathcal{V}(P)=\left(v_{1}, \ldots, v_{j}\right)$, and $\mathcal{A}(P)=\left(a_{1}, \ldots, a_{i+j-1}\right)$ and there is no $P^{\prime} \in \mathcal{D} E C$ with $\mathcal{H}\left(P^{\prime}\right)=\mathcal{H}(P), \mathcal{V}\left(P^{\prime}\right)=\mathcal{V}(P)$, and $\mathcal{A}\left(P^{\prime}\right)=\mathcal{A}(P)$ such that the SCDRs of $P^{\prime}$ are connected to each other with their bottom left and upper right corners then $P$ is the $N W$-component of $F$. If no such polyomino exists then $F$ does not have a $N W$-component.

Proof. Define a set by $T=\bigcup_{k=1}^{i+j-1} A_{k}$ and let $Q^{\prime}=F \cap T$. Since $\mathcal{A}(P)=$ $\left(a_{1}, \ldots, a_{i+j-1}\right)$ we get $Q^{\prime}$ by applying some (possibly none) antislides on $P$. Let $Q$ be an arbitrary discrete set with the projections $\mathcal{H}(Q)=\left(h_{1}^{q}, \ldots, h_{m}^{q}\right)$, $\mathcal{V}(Q)=\left(v_{1}^{q}, \ldots, v_{n}^{q}\right)$, and $\mathcal{A}(Q)=\left(a_{1}^{q}, \ldots, a_{m+n-1}^{q}\right)$ that we get by applying some antislides on $P$. This time we allow that some of the coordinates of $\mathcal{H}(Q)$ and $\mathcal{V}(Q)$ are zero. Clearly, $a_{l}^{q}=a_{l}$ for each $l=1, \ldots, i+j-1$ and $Q \subseteq T$. Moreover, for the horizontal and vertical projections of $Q$ exactly one of the following cases holds

(i) $\exists i^{\prime} \leq i$ such that $h_{i^{\prime}}^{q} \neq h_{i^{\prime}}$ or $\exists j^{\prime} \leq j$ such that $v_{j^{\prime}}^{q} \neq v_{j^{\prime}}$,

(ii) $h_{i^{\prime}}^{q}=h_{i^{\prime}}$ for each $i^{\prime}=1, \ldots, i$ and $v_{j^{\prime}}^{q}=v_{j^{\prime}}$ for each $j^{\prime}=1, \ldots, j$.

Assume that Case (i) is true with $h_{i^{\prime}}^{q} \neq h_{i^{\prime}}$ for an $i^{\prime} \leq i$. Then, there also exists an $i^{\prime \prime} \leq i$ such that $h_{i^{\prime \prime}}^{q}>h_{i^{\prime \prime}}$ or a $j^{\prime \prime} \leq j$ such that $v_{j^{\prime \prime}}^{q}>v_{j^{\prime \prime}}$. Clearly, in this case there is no discrete set $F^{\prime}$ with the projections $\mathcal{H}\left(F^{\prime}\right)=\left(h_{1}, \ldots, h_{m}\right)$ and $\mathcal{V}\left(F^{\prime}\right)=\left(v_{1}, \ldots, v_{n}\right)$ such that $F^{\prime} \cap T=Q$. Assuming that Case (i) is true with $v_{j^{\prime}}^{q} \neq v_{j^{\prime}}$ for a $j^{\prime} \leq j$ we get the same in a similar way. Therefore $F$ can have the given projections if and only if for $Q^{\prime}$ Case (ii) is true which is possible only if $Q^{\prime} \subseteq\{1, \ldots, i\} \times\{1, \ldots, j\}$. Since $\mathcal{H}\left(Q^{\prime}\right)=\left(h_{1}, \ldots, h_{i}\right)$ and $\mathcal{V}\left(Q^{\prime}\right)=\left(v_{1}, \ldots, v_{n}\right)$ it follows that $F$ can have the prescribed projections if and only if $F \cap E=\emptyset$ where $E$ is defined by (11). Then, $\{1, \ldots, i\} \times\{1, \ldots, j\}$ is the SCDR of a set $G \subseteq F$ consisting of one or more components of $F$. However, if $G$ consists of several components of $F$ then $G \in \mathcal{D} E C$ based on Lemma 1 and the SDCRs of the components of $G$ are connected to each other with their bottom left and upper right corners on the basis of Lemma 2 which is a contradiction. Consequently, $G$ is a simple polyomino. Since $P$ satisfies the conditions which guarantees that property $(\alpha)$ holds $G=P$ and so the first part of the theorem is proven. The second part of the theorem follows from the fact that the position that satisfies the necessary conditions of Theorem 1 is uniquely determined.

Similar theorems can be given for testing the existence of NE-, SE-, and SW-components. We now can outline an algorithm for reconstructing decomposable discrete sets with given horizontal, vertical, diagonal, and antidiagonal projections. 


\section{Algorithm 4-DEC}

Input: the vectors $H \in \mathbb{N}^{m}, V \in \mathbb{N}^{n}, D \in \mathbb{N}_{0}^{m+n-1}$, and $A \in \mathbb{N}_{0}^{m+n-1}$. Output: the uniquely determined decomposable discrete set with projections $H, V, D$, and $A$ or FAIL (if no such set exists).

1: repeat

try to reconstruct a NW-component by identifying its SCDR and using the corresponding elements of the vectors $H, V$, and $A$;

if not succeed then try to reconstruct a NE-component by identifying its SCDR and using the corresponding elements of the vectors $H, V$, and $D$; if not succeed then try to reconstruct a SE-component by identifying its SCDR and using the corresponding elements of the vectors $H, V$, and $A$; if not succeed then try to reconstruct a SW-component by identifying its SCDR and using the corresponding elements of the vectors $H, V$, and $D$; if not succeed then break;

modify $H, V, D$, and $A$ according to the reconstructed component and in the following omit the reconstructed part of the discrete set;

until all the components are reconstructed;

2: if the diagonal/antidiagonal projection is not equal to the given vector $D / A$, respectively then

$\left\{\right.$ let $P_{1}, \ldots, P_{l}$ denote the polyominoes reconstructed in Step 1; $i=0$;

\section{repeat}

assuming that $P_{1}, \ldots, P_{i}$ are components try to decompose further components in order SW, SE, NE, NW similarly as in Step 1; $i=i+1$;

until all the components are reconstructed or $i=l ;\}$

3: if the diagonal/antidiagonal projection is not equal to the given vector $D / A$, respectively then FAIL (no solution);

Turning to the analysis of the algorithm we can say the following

Theorem 3. Algorithm 4-DEC solves the problem 4 -RECONSTRUCTION $(\mathcal{D} E C)$. Assume that the reconstructed set consists of components $F_{1}, \ldots, F_{k}$ and let $C_{i}$ denote the time complexity of reconstructing the component $F_{i}(i=1, \ldots, k)$. Then, the worst case time complexity of the algorithm is of $k \cdot \max _{1 \leq i \leq k} C_{i}$ which is polynomial. The solution is uniquely determined.

Proof. As a straight consequence of the algorithm we get that the reconstructed set is decomposable and has the given projections. Assuming that the $l$-th $(l=1, \ldots, k)$ component to be reconstructed is a NW-component it takes $O(m+n)$ time to find the (uniquely determined) position which satisfies the necessary conditions of Theorem 1. We do it simply by scanning the vectors $\widetilde{H}$ and $\widetilde{V}$. In order to test whether this position is the bottom right position of the SCDR of the NW-component we try to reconstruct this component based on Theorem 2 which takes $C_{l}$ time. The same is true if the $l$-th component is a NE-, $\mathrm{SE}$ - or SW-component. In the worst case the component is a SW-component, 
i.e., we try to reconstruct the $l$-th component at most four times and so the reconstruction complexity of Step 1 is $\max _{1 \leq i \leq k} C_{i}$ which is polynomial because of property $(\alpha)$. Theorem 2 guarantees the existence of a NW-component only when there is no decomposable discrete set with the same horizontal, vertical, and antidiagonal projections such that the components are connected to each other with their bottom left and upper right corners. Therefore it can occur that we accept the reconstructed polyomino as a NW-component although the decomposable discrete set to be reconstrcuted has no NW-component at all. The same is true for NE-, SE-, and SW-components, too. These situations result that the algorithm cannot reconstruct the decomposable discrete set with the given projections in Step 1. However, it reconstructs some polyominoes $P_{1}, \ldots, P_{l}$ such that there exists an $l^{\prime} \leq l$ for which $P_{1}, \ldots, P_{l^{\prime}-1}$ are components and $P_{l^{\prime}}$ is not a component of the decomposable discrete set. Then, the components $F_{1}, \ldots, F_{l^{\prime}-1}$ are already reconstructed and the remaining components of the discrete set can be reconstructed in reversed order in Step 2. If $l^{\prime}$ is known then all the remaining components can be reconstructed in Step 1. Since $l^{\prime}$ is not known in Step 2 we have to call Step 1 at most $k$ times and so the reconstruction complexity of Step 2 is of $k \cdot \max _{1 \leq i \leq k} C_{i}$ in the worst case. The uniqueness of the solution follows from property $(\alpha)$.

\section{Reconstruction of $h v$-Convex 8- but Not 4-Connected Discrete Sets from Four Projections}

The class of $h v$-convex 8 - but not 4 -connected discrete sets (denoted by $\mathcal{S}_{8}^{\prime}$ ) was introduced in 2 . In the same paper the authors gave a reconstruction algorithm in this class using the horizontal and vertical projections. This algorithm has worst case time complexity of $O(m n \cdot \min \{m, n\})$ and the solution is not always uniquely determined. Then, in [3] it is shown that using also the diagonal and antidiagonal projections the algorithm can be speeded up having complexity of $O(m n)$ and in this case uniqueness also holds. In the following we show that this is a consequence of

Theorem 4. $\mathcal{S}_{8}^{\prime} \subseteq \mathcal{D} E C$.

Proof. Let $F \in \mathcal{S}_{8}^{\prime}$. Then, clearly, the number of components of $F$ is at least 2. Since $F$ is $h v$-convex the sets of the row/column indices of the components consist of consecutive integers and they are disjoint. Then, property $(\beta)$ is satisfied. Moreover, on the basis of Theorem 5 in 3] and Theorem 3 in [18 the components can be reconstructed uniquely from the horizontal and vertical projections in $O(m n)$ time, i.e., property $(\alpha)$ also holds. Finally, the configuration of the components can follow only two cases (see Theorem 2 in [3]). Namely, the SCDRs of the components are connected to each other with their bottom right and upper left or with their bottom left and upper right positions (see Fig. 4a and $4 \mathrm{~b}$, respectively). Clearly, both configurations are decomposable.

Then, applying Theorem 3 we get the following 


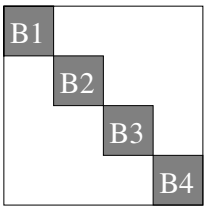

(a)

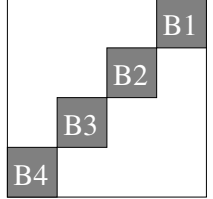

(b)

Fig. 4. The two possible configurations of the components in the class $\mathcal{S}_{8}^{\prime}$

Corollary 1. Algorithm 4-DEC solves the problem 4 -RECONSTRUCTION $\left(\mathcal{S}_{8}^{\prime}\right)$ in $O(m n)$ time. The reconstructed set is uniquely determined.

\section{Conclusions and Further Work}

In this paper we have introduced a new class of discrete sets, the class of decomposable discrete sets and we have given a reconstruction algorithm in this class using four projections. It is shown that the algorithm has polynomial time complexity. Then, it is proven that the class of $h v$-convex 8 - but not 4connected sets is a subclass of $\mathcal{D} E C$. As a consequence we got that the problem 4$\operatorname{Reconstruction}\left(\mathcal{S}_{8}^{\prime}\right)$ can be solved in $O(m n)$ time. Since the complexity of our algorithm strongly depends on the fact that the components are uniquely determined by the horizontal and vertical projections it seems to be important to find classes of discrete sets where the reconstruction problem can be solved uniquely.

It is shown that in some cases the discrete set can be decomposed along the diagonal and antidiagonal projections to facilitate the reconstruction. However, in some cases the decomposition into components is impossible. For example, the configuration in Fig. 2 ean be decomposed into two parts (one containing $B 1$ and $B 2$, and the other containing $B 3$ and $B 4$ ) by the antidiagonal projection but then, the two parts cannot be further decomposed into components since the diagonal projections of the two parts are not independent. In some unfortunate cases the components cannot be separated at all (see, e.g., Fig. 2b and 2h). If the set is not decomposable then our algorithm simply FAILs without giving a solution. It is an interesting question whether it could be decided in advance if a discrete set is decomposable. Further investigation of the undecomposable configurations is also needed.

Throughout the paper it was assumed that every coordinate of the horizontal and vertical projections is nonzero, i.e., $h_{i}>0$ and $v_{j}>0$ for all $i \in\{1, \ldots, m\}$ and $j \in\{1, \ldots, n\}$. However, the results can be generalized easily to handle decomposable discrete sets where some of the coordinates of the horizontal or vertical projections are zero. In our work we concentrated on discrete sets consisting of components which satisfy some special properties (namely, properties $(\alpha)$ and $(\beta))$. More work has to be done on the field whether assuming weaker properties about the components the reconstruction process remains tractable. Further work in this field can lead us towards designing efficient reconstruction algorithms for important classes like the one of $h v$-convex sets. 


\section{References}

1. P. Balázs, Reconstruction of decomposable discrete sets from four projections, Technical Report at the University of Szeged (2004) http://www.inf.u-szeged.hu/ pbalazs/research/research.html

2. P. Balázs, E. Balogh, A. Kuba, A fast algorithm for reconstructing $h v$-convex 8connected but not 4-connected discrete sets, Lecture Notes in Computer Science 2886 (2003) 388-397.

3. P. Balázs, E. Balogh, A. Kuba, Reconstruction of 8-connected but not 4-connected $h v$-convex discrete sets, Discrete Applied Mathematics, accepted.

4. E. Balogh, A. Kuba, Cs. Dévényi, A. Del Lungo, Comparison of algorithms for reconstructing $h v$-convex discrete sets, Lin. Alg. and Its Appl. 339 (2001) 23-35.

5. E. Barcucci, A. Del Lungo, M. Nivat, R. Pinzani, Reconstructing convex polyominoes from horizontal and vertical projections, Theor. Comput. Sci. 155 (1996) 321-347.

6. R.A. Brualdi, Matrices of zeros and ones with fixed row and column sum vectors, Lin. Algebra and Its Appl. 33 (1980) 159-231.

7. S. Brunetti, A. Daurat, Reconstruction of discrete sets from two or more X-rays in any direction, Proceedings of the seventh International Workshop on Combinatorial Image Analysis (2000) 241-258.

8. M. Chrobak, Ch. Dürr, Reconstructing $h v$-convex polyominoes from orthogonal projections, Information Processing Letters 69(6) (1999) 283-289.

9. A. Daurat, Convexité dans le plan discret. Application à la tomographie, Thèse de doctorat de l'Université Paris 7 (2000) http://llaic3.u-clermont1.fr/ daurat/these.html

10. A. Del Lungo, Polyominoes defined by two vectors, Theor. Comput. Sci. 127 (1994) 187-198.

11. A. Del Lungo, M. Nivat, R. Pinzani, The number of convex polyominoes reconstructible from their orthogonal projections, Discrete Math. 157 (1996) 65-78.

12. R.J. Gardner, P. Gritzmann, Uniqueness and complexity in discrete tomography, In 15. (1999) 85-113.

13. L. Hajdu, R. Tijdeman, Algebraic aspects of discrete tomography, Journal für die reine und angewandte Mathematik $\mathbf{5 3 4}$ (2001) 119-128.

14. G.T. Herman, A. Kuba (Eds.), Discrete Tomography, Special Issue. Int. J. Imaging Systems and Techn. 9 (1998) No. 2/3.

15. G.T. Herman, A. Kuba (Eds.), Discrete Tomography: Foundations, Algorithms and Applications (Birkhäuser, Boston, 1999).

16. A. Kuba, The reconstruction of two-directionally connected binary patterns from their two orthogonal projections, Comp. Vision, Graphics, and Image Proc. $\mathbf{2 7}$ (1984) 249-265.

17. A. Kuba, Reconstruction in different classes of $2 \mathrm{D}$ discrete sets, Lecture Notes on Computer Sciences 1568 (1999) 153-163.

18. A. Kuba, E. Balogh, Reconstruction of convex 2D discrete sets in polynomial time, Theor. Comput. Sci. 283 (2002) 223-242.

19. H.J. Ryser, Combinatorial properties of matrices of zeros and ones, Canad. J. Math. 9 (1957) 371-377.

20. P. Soille, From binary to grey scale convex hulls, Fundamenta Informaticae 41 (2000) 131-146.

21. G.W. Woeginger, The reconstruction of polyominoes from their orthogonal projections, Inform. Process. Lett. 77 (2001) 225-229. 